This is an Open Access article, distributed under the terms of the Creative Commons Attribution licence (http://creativecommons.org/licenses/by/4.0/), which permits unrestricted re-use, distribution, and reproduction in any medium, provided the original work is properly cited.

doi:10.1017/jfm.2016.672

\title{
Droplet impact onto moving liquids
}

\section{J. Rafael Castrejón-Pita ${ }^{1} \dagger$, Beatriz N. Muñoz-Sánchez ${ }^{2}$, Ian M. Hutchings ${ }^{2}$ and A. A. Castrejón-Pita ${ }^{3}$}

\author{
${ }^{1}$ School of Engineering and Material Science, Queen Mary, University of London, \\ London E1 4NS, UK \\ ${ }^{2}$ Department of Engineering, University of Cambridge, Cambridge CB3 OFS, UK \\ ${ }^{3}$ Department of Engineering Science, University of Oxford, Oxford OX1 3PJ, UK
}

(Received 1 February 2016; revised 6 July 2016; accepted 11 October 2016; first published online 15 November 2016)

From rain drops landing on the ocean to inkjet printing, the impact of droplets onto moving liquid surfaces is a ubiquitous process in nature and in industry. A rich range of phenomena can arise. The behaviour depends on the inertia, the properties of the drops and the relative speeds in the impact zone. While the result ranges from tranquil coalescence to violent splashing, intermediate regimes also occur, including partial and complete bouncing and even 'surfing' of the droplet. These regimes are determined by the ratio of the drop and surface velocities and the liquid properties. A regime diagram can be constructed in which distinct dynamical regimes are clearly identified.

Key words: breakup/coalescence, drops, drops and bubbles

\section{Introduction}

The impact of droplets onto moving liquid surfaces and films occurs frequently in nature, and is critically important in many industrial processes, from spray painting and crop spraying, to inkjet printing. It is probably the latter that has attracted the most recent interest, as it is capable of delivering precise amounts of material in the form of liquid droplets under digital control. Inkjet methods are not only being increasingly used for graphical printing, but are also central to some types of '3D printers', which aim to manufacture products that cannot be produced by any other method. For the continuous development of inkjet, and in order to overcome current limitations, a better understanding of droplet formation, droplet-substrate interaction, pinning phenomena, drop coalescence and material compatibility is needed (Eggers \& Villermaux 2008; Derby 2010).

The detailed study of droplets impacting an initially stationary pool of the same liquid began in the 19th century with the work of Worthington (1908), which continues to be an active area of research today. The recent work by Castrejón-Pita, Castrejón-Pita \& Hutchings (2012) and Thoraval et al. (2012) have shown that the dynamics at the interface is complex, with a diversity of phenomena being observed 
ranging from the trapping of air bubbles to the shedding of vortices (Thoroddsen 2002; Deegan, Brunet \& Eggers 2008; Thoroddsen, Etoh \& Takehara 2008). These effects are influenced by the liquid properties and the droplet speed: a slowly moving droplet of a highly viscous liquid penetrates smoothly into the pool, whereas a faster, less viscous droplet can splash, generating vortices at the interface (Moore et al. 2014) and a plethora of daughter microdroplets. Studies of droplet impact onto static solid surfaces have also shown a wide variety of behaviour, from droplets bouncing on hydrophobic surfaces (Bird et al. 2013), to the dependence of droplet splashing on the roughness of the surface (Xu, Barcos \& Nagel 2007) and on the surrounding gas pressure $(\mathrm{Xu}$, Zhang \& Nagel 2005). While the majority of these studies have focused on the impact of droplets onto stationary substrates, other conditions found in nature and in commercial applications involve the impact of drops onto moving substrates, e.g. rain droplets falling on moving bodies of water, high-speed inkjet printing or spraying/painting onto moving substrates which may be dry or already wet (Derby 2010). Experimental and theoretical studies of liquid impact onto moving solid substrates are very scarce, but have demonstrated that splashing or bouncing can be controlled, and even partially suppressed, by adjusting the speed of the substrate (Bird, Tsai \& Stone 2009; Lhuissier et al. 2013). On the other hand, from the theoretical point of view, some light has been shed on related splashing problems such as angled impact of solid bodies onto liquid surfaces or oblique water-entry events, where asymptotic theories may be applicable to the problem at hand (Korobkin 1988; Howison, Ockendon \& Oliver 2004; Moore et al. 2012; Sun \& Wu 2013).

Whether an impacting droplet splashes after impact on a solid substrate is controlled by the dynamics of the lamella (the liquid sheet formed after impact) relative to the target surface (Bird et al. 2009). Previous studies on moving solid substrates have determined that the splashing number $K$, defined as $K=W e \sqrt{R e}$, is an appropriate parameter to categorise the resulting dynamics, which in turn depends on two other dimensionless groups: the Reynolds $(R e)$ and the Weber $(W e)$ numbers. $R e=\rho r v_{n} / \mu$ and $W e=\rho r v_{n}^{2} / \sigma$ respectively, where $\mu, \rho$ and $\sigma$ are the viscosity, density and surface tension of the liquid and $v_{n}$ and $r$ are the impact speed and radius of the droplet (Thoraval et al. 2012). The Reynolds and Weber numbers quantify the ratios of inertial forces to viscous and surface tension forces respectively. In other words, these dimensionless numbers describe the relative importance of the impact inertia over the viscous and surface forces which tend to decelerate the liquid or breakup the expanding liquid rim formed after impact. In this scenario, the critical value of $K$ depends on the ratio between the tangential and the impact velocity (Bird et al. 2009).

While the impact of droplets onto moving solid substrates has been successfully studied in recent years, the impact onto moving liquids has remained largely unexplored due primarily to the considerable practical difficulties involved. These include the generation of uniformly moving liquid pools, the controlled production of single droplets of various sizes and properties and the implementation of high-speed imaging set-ups that visualise the impact region from either the droplet or the pool frame of reference. This manuscript presents an experimental arrangement which overcomes these limitations and investigates the impact of droplets onto moving pools of the same liquid. We report the existence of four distinct regimes of behaviour, identified as: droplet surfing, smooth coalescence, lamella jetting and splashing. We find that these regimes are well grouped and determined by the initial dynamic conditions and the properties of the fluid. The regimes of lamella jetting 
and splashing present features previously unreported. Experiments show that the dimensionless groups, previously known to regulate the splashing behaviour on static substrates, when defined appropriately in terms of the droplet speed relative to the liquid substrate, also govern the impact dynamics here.

\section{Experimental set-up}

A schematic view of our experimental set-up is shown in figure 2. Our experiments involved the careful production and visualisation of single droplets of water-glycerol mixtures, from 1.0 to $2.2 \mathrm{~mm}$ in radius, which travelled vertically downwards onto a pool of the same liquid moving at a constant speed at right angles to the motion of the impacting droplet. Single droplets of different sizes and speeds were formed either by dripping or jetting and the conditions were controlled by adjusting the dripping flow, the jetting characteristics and/or the nozzle size. The moving liquid pool was formed by rotating the annular channel about a horizontal axis. The liquid pool is contained in a rotating annular channel with an internal radius of $70.0 \mathrm{~mm}$ and width of $20.0 \mathrm{~mm}$ mounted on the shaft of a DC electric motor. The rotation frequency was monitored by a laser-photodiode pair, allowing the frequency to be controlled within $\pm 0.02 \mathrm{~Hz}$. The tangential relative speed of the liquid surface $\left(v_{t}\right)$ at the point of drop impact was therefore controlled by varying the rotation frequency. The channel was constructed of transparent acrylic in order to permit visualisation. Once the system was set in motion and reached the desired rotation rate, the channel was filled with the working fluid with the help of a small funnel or a pipette. The depth of the pool was adjusted by carefully adding liquid without splashing droplets onto the walls of the channel which could obstruct the visualisation. Great care was also taken to avoid introducing bubbles to the liquid pool or onto the channel walls, which would otherwise interfere with the visualisation.

The fluid depth of $6.0 \mathrm{~mm}$ was kept constant throughout all the experiments. A spin-up time of at least $10 \mathrm{~min}$ was allowed before performing the drop impact experiments. This proved to be enough for any surface irregularities to die out; solid-body rotation was assumed in all our experiments. Practical limitations restricted our studies to rotational frequencies within the range of $4.0-25.5 \mathrm{~Hz}$. Below this range the centripetal force is not enough to overcome gravity and retain the liquid within the channel, whereas above this range the rotation of the motor was found to be unstable. Impacting drops were produced by two methods: (i) simple dripping from polymethylpentene nozzles at various heights for experiments with low impact velocities and large radius and (ii) by means of a large-scale drop on demand generator. This large-scale system is sketched in figure 3 and is a modification of the one described in Castrejón-Pita et al. (2011). The drop generator was developed to achieve the higher impact velocities. In brief, the system uses an electromagnetic actuator (a shaker) to push liquid out of a nozzle of $2.0 \mathrm{~mm}$ diameter. A rubber membrane transmits single pressure pulses produced by the actuator to the liquid reservoir within the system. Droplet speeds in the range from 1.2 to $1.7 \mathrm{~m} \mathrm{~s}^{-1}$ were produced by this method. The working fluids consisted of water-glycerol mixtures, with Newtonian viscosities ranging from 1 to $200 \mathrm{mPa}$ s and surface tension values in the range of 61.9 to $70.8 \mathrm{mN} \mathrm{m}^{-1}$. Experimental conditions were adjusted to produce near-spherical drops with shape aspect ratios $>0.9$. All the experiments were performed at $21.0 \pm 0.2^{\circ} \mathrm{C}$ under typical atmospheric conditions with air as the surrounding fluid. Fluid density, viscosity and surface tension were measured with an Anton Paar DMA 35N density meter, a Hydramotion 700 Viscolite viscometer and a SITA bubble pressure T-15 tensiometer, respectively. 
(a)

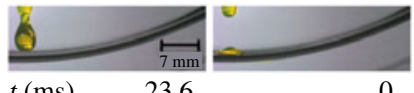

(b)
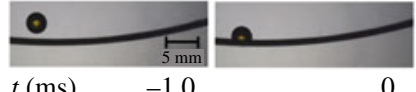

(c)

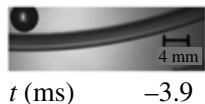

(d)

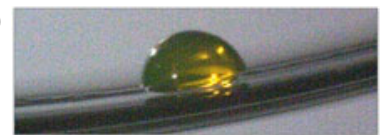

(e)

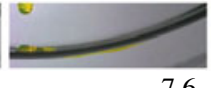

7.6

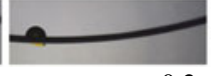

0.2
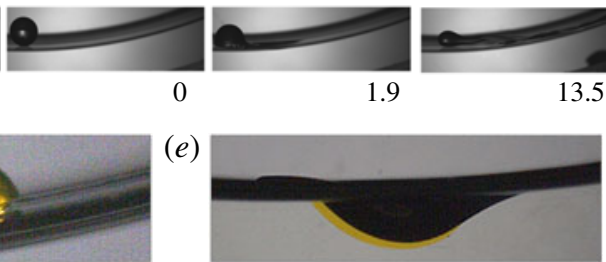
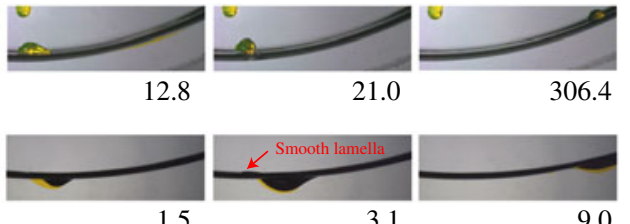

1.5

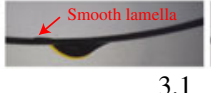

3.1
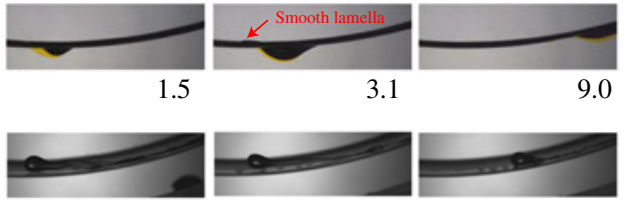

13.5
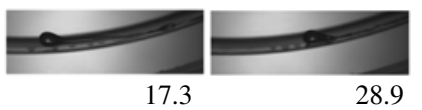

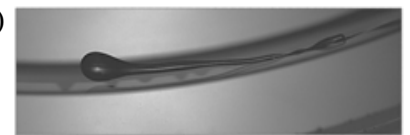

FIGURE 1. (Colour online) Impact behaviour for three different conditions. (a) Droplet surfing: a droplet (viscosity $100 \mathrm{mPa}$ s) gently deposited at a speed of $0.06 \mathrm{~m} \mathrm{~s}^{-1}$ onto a deep pool of the same liquid travelling from left to right at $1.60 \mathrm{~m} \mathrm{~s}^{-1}$. The droplet partially coalesces within the first $7 \mu \mathrm{s}$ but then a stable air film is formed between the liquid surfaces which prevents further coalescence and the droplet rides over the surface ('surfing'). (b) Smooth coalescence: a droplet at $1.36 \mathrm{~m} \mathrm{~s}^{-1}$ impacts a liquid surface (viscosity $200 \mathrm{mPa} \mathrm{s}$ ) travelling at $4.16 \mathrm{~m} \mathrm{~s}^{-1}$. The droplet smoothly penetrates and coalesces with the pool of liquid without splashing, and very little mixing occurs. (c) Lamella jetting: a droplet moving at $0.45 \mathrm{~m} \mathrm{~s}^{-1}$ impacts a liquid surface of the same liquid (viscosity $100 \mathrm{mPa} \mathrm{s}$ ) moving at $4.10 \mathrm{~m} \mathrm{~s}^{-1}$. The droplet initially coalesces with the pool and an air film is formed but is not stable. The droplet remains connected to the pool by a filament and progressively coalesces with the pool without splashing. Images $(d-f)$ show frames from the sequences in $(a-c)$ at higher magnification. See also supplementary movies 1, 2, 3 and 5 available online at https://doi.org/10.1017/jfm.2016.672.

Grey scale and colour high-speed imaging were employed to capture the dynamics above and below the droplet-pool interface during impact and to monitor mixing at the interface. Grey scale shadowgraphy allowed for a clear determination of the impact dynamics over the surface of the liquid pool, whilst colour imaging and laser fluorescence permitted us to extract detailed information of the internal dynamics of the impact. A combination of these techniques allowed the visualization of the impacting events, i.e. the general form of the flow and the internal dynamics of the drop and liquid pool. Figure 2 shows the position of the lamp for shadowgraphy (an example of this type of visualisation is shown in figure 1c) and also illustrates the use of the laser sheet for fluorescence imaging. In contrast, colour imaging required front illumination, where the lamp was placed oblique to the camera and pointing towards the impact point (examples of this type of visualisation are shown in figure $1 a, b)$. Some additional experiments were performed combining front illumination and laser fluorescence to visualise the full dynamics under and inside the drop and above the pool, an example of this visualisation is shown in figure 4. The shadowgraphy system comprised a PhotoFluor II lamp coupled to a liquid-fibre optical guide and an optical diffuser for backlight illumination. The same lamp was used for front illumination to record in colour. For colour imaging and laser fluorescence visualisation, small quantities of sodium fluorescein $(<0.02 \mathrm{wt}$.\% concentration) were added to the fluids used to produce the drops. Within experimental error, no changes in the viscosity, density or surface tension resulted from the addition of this dye 


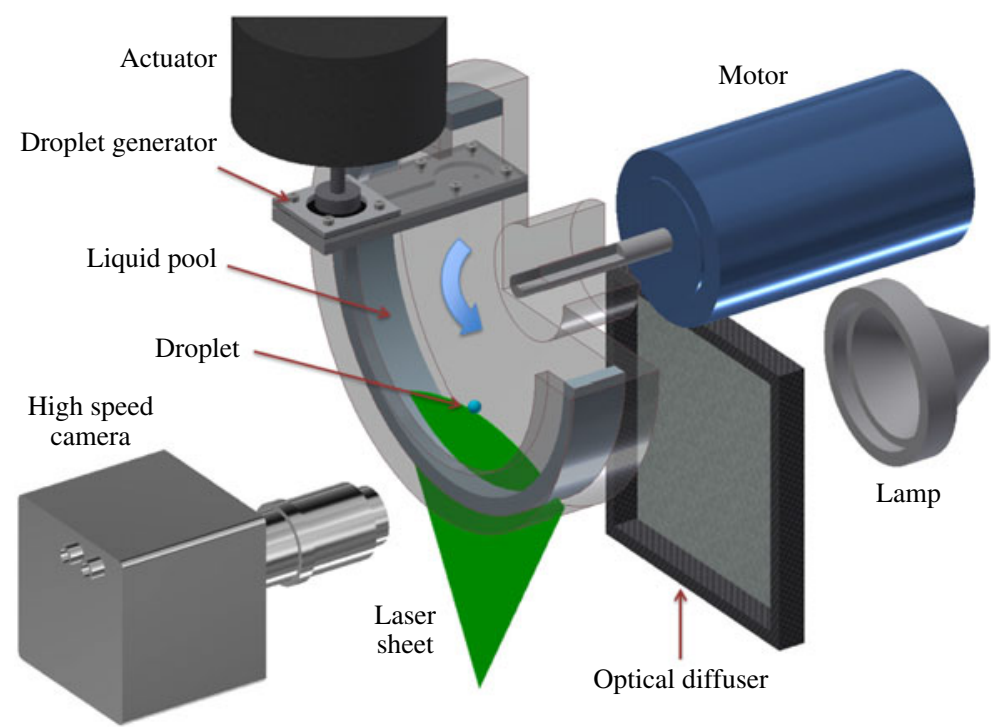

FIGURE 2. (Colour online) Schematic view of the experimental set-up including a cutaway view of the liquid channel. Both the impacting droplet and the liquid pool are of the same fluid.

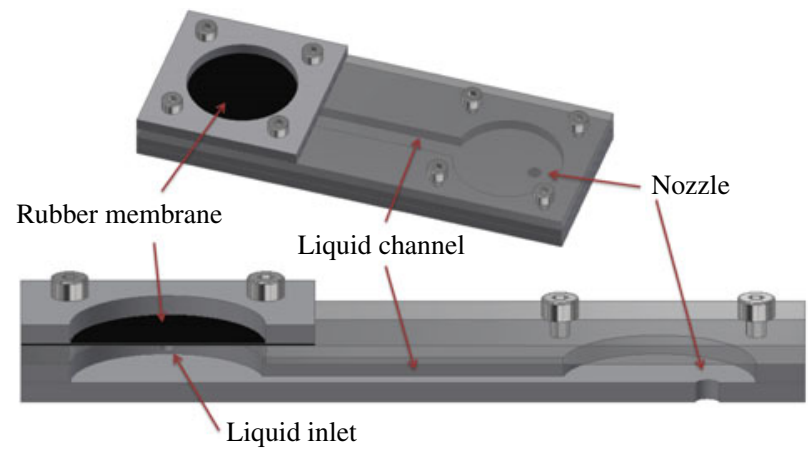

FIGURE 3. (Colour online) Schematic view and cutaway view of the droplet generator device. The system is a modified version of the device presented in Castrejón-Pita $e t$ al. (2011).

(Castrejón-Pita et al. 2012). A $532 \mathrm{~nm} 500 \mathrm{~mW}$ continuous (diode) laser coupled to a cylindrical lens was used to produce a $<100 \mu \mathrm{m}$-thick sheet of illumination, which was directed from below towards the centre of the impacting zone and perpendicular to the camera view in order to visualise the contact line and the drop's internal dynamics under the surface of pool (Castrejón-Pita et al. 2012). Two high-speed cameras were used; a monochrome Phantom V310 for fast dynamics and small droplets and a Phantom V710 for slow dynamics, large droplets and laser/colour visualisation. Optical resolutions ranged from 18 to $53 \mu \mathrm{m} \mathrm{pixel}^{-1}$ with either a microscope lens (Navitar $12 \times$ UltraZoom) or a Tamron AF90 macro lens. An image analysis routine written in Matlab was utilised to extract the droplet impact speed and radius for each experiment. The impact behaviour was explored by a parametric 


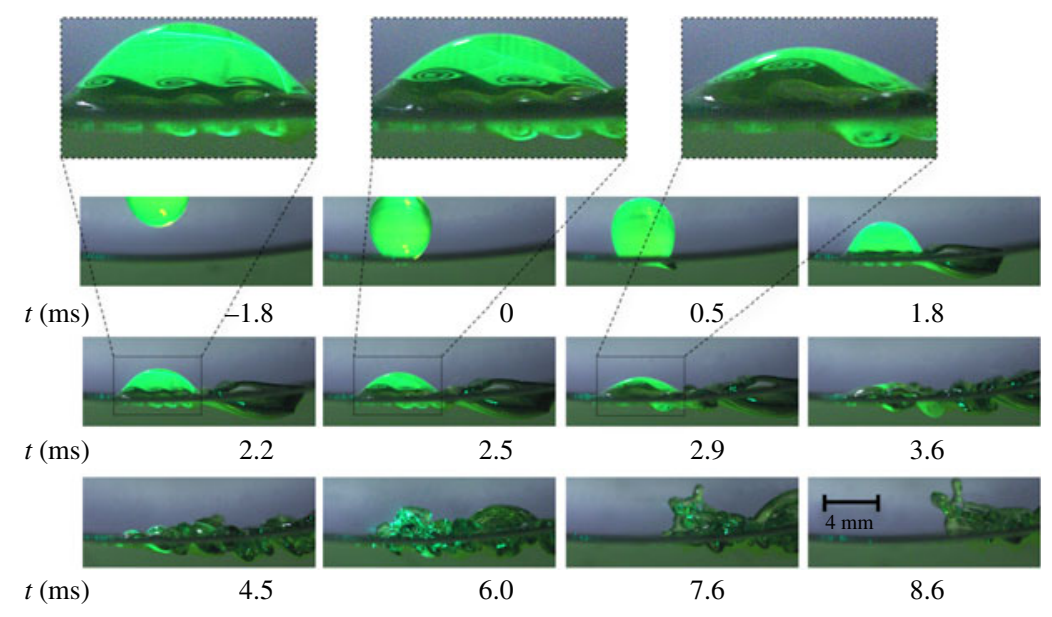

FIgURE 4. (Colour online) Time evolution of the impact of a droplet with viscosity $5 \mathrm{mPa} \mathrm{s}$ at a normal speed of $1.3 \mathrm{~m} \mathrm{~s}^{-1}$ onto a pool of the same liquid moving from left to right at $3.3 \mathrm{~m} \mathrm{~s}^{-1}$. Laser and colour high-speed imaging were combined to observe the mixing process; the impacting droplet contained $0.02 \%$ fluorescein. See also supplementary movie 4.

study of fluid properties and impact conditions and a regime diagram was constructed which classifies the different behaviours observed. A typical experimental routine consisted of keeping the rotation constant while varying the impact speed of the drop. The rotation rate was then increased and a new series of experiments carried out. On average, a single set of conditions was explored per day.

\section{Results}

Examples of impact behaviour are shown in figures 1 and 4. The range of fluid properties and impacting speeds used in these experiments, once converted into dimensionless form, are well within the printability regime (i.e. where inkjetted droplets are known to occur) according to Derby (2010). Outside this range, surface tension or viscous effects are too strong to permit the production of individual droplets. The range of ratios between the tangential and normal speeds explored in this work were from 1.5 to 48.5 .

Our experiments show that the impact behaviour could be classified into four different regimes: surfing, smooth coalescence, lamella jetting and splashing.

The first regime, surfing, is commonly found in higher viscosity liquids, low to medium impact speeds and high rotational velocities. Under this behaviour the impacting drop never fully penetrates the liquid pool, but bounces without coalescing with the pool and remains levitated by a thin film of air, just above the surface (figure 1a). As this occurs, the drop moves slowly across the liquid surface in the direction of rotation of the pool, oscillating back and forth before reaching an equilibrium point, where it remains in a stable state, with the drag forces balancing the tangential component of its weight. Occasionally, slight loss of liquid from the drop occurs on impact, which becomes incorporated into the moving pool. Surfing can also be observed at low impact speeds (inside the smooth coalescence regime), as the drop starts to coalesce the relatively high speed of the pool induces quick breakup 


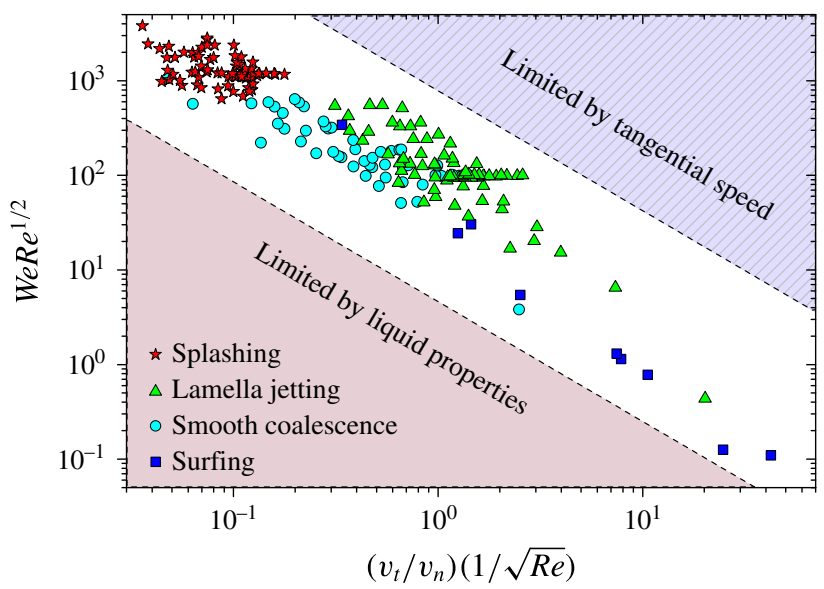

FIgURE 5. (Colour online) Regime diagram showing the different impact dynamics in terms of relevant dimensionless numbers for glycerol-water mixtures over a wide range of impact conditions.

of the drop, with part of it being left behind, surfing on the pool. Finally, surfing is also found at higher impact and rotational speeds (near the lamella jetting regime), whereby upon impact, part of the original drop coalesces with the liquid pool, whilst the other part breaks up and remains surfing on the surface. A similar scenario has been previously observed by Thoroddsen \& Mahadevan (1997) in experiments with rapidly rotating drums partly filled with high viscosity liquids rotating at $8 \mathrm{~Hz}$. There, air entrainment produced the shedding of tiny drops that remained hydroplaning over a thin layer of rapidly moving fluid.

In conditions of smooth coalescence, seen as light blue circles in figure 5, the drop penetrates the pool smoothly and rapidly, with all of its volume fully merging with the pool. In this case, little mixing is observed at the interface and a clearly visible smooth lamella is often formed opposite to the direction of motion (indicated in figure $1 b$ ). The observed depth reached by the drop upon impact depended on the absolute velocity. The maximum penetration depth is therefore found for low rotation frequencies and high impact speeds. The maximum penetration depth observed in our experiment was approximately half of the total depth of the pool, ensuring that wall effects are kept to a minimum.

In lamella jetting, seen as green triangles in figure 5, the drop partially coalesces at the first point of contact, but not all its volume merges into the pool (figure $1 c$ ). A large proportion of the drop remains surfing over the surface (or bounces back after the initial impact) and is dragged by the moving pool beyond the original impact point. A liquid filament is formed between the droplet and the pool, which remains connecting them, even if the droplet lifts and separates from the moving surface (figure $1 f$ ). The fluid in the drop continues to coalesce with the substrate through the ligament.

The splashing regime is identified by the red stars in figure 5. In this regime the rear part of the impacting drop rapidly coalesces with the pool while a lamella emerges from the front of the drop. The inertia of the drop overcomes viscosity and surface tension, which leads to the breakup of the rim of the lamella into droplets. As observed in the upper images of figure 4, in the splashing regime, vortices are shed at the drop-pool contact line within the first moments of contact. The train of 
vortices seen within the drop is an optically (distorted) refracted/reflected image of the contact line (or meniscus) of the impact zone. The view is distorted due to the shape of the drop/pool system and the difference of refractive index between air and the liquid. The vortices in this chain of vortices all have the same sign, pointing towards a Kelvin-Helmholtz-type shear-driven instability, i.e. a high shear between the drop and the pool at the point of contact. This is in contrast with the von Kármán vortex street (of alternating sign) observed by Castrejón-Pita et al. (2012) and Thoraval et al. (2012, 2013). Vortex shedding was only observed in the splashing regime.

Previous studies of drop impact onto stationary liquid films have established that the splashing parameter $K$, which encompasses all the drop properties, is appropriate to differentiate the splashing behaviour of the impact (Josserand \& Zaleski 2003; Thoraval et al. 2012). In fact, according to Bird et al. (2009) the splashing parameter also controls the dynamics of drops impacting moving solid (dry) substrates. In addition, some recent studies carried out by Riboux \& Gordillo (2014) and Liu, Tang $\& \mathrm{Xu}$ (2015) have suggested that the surrounding gas also plays a role in regulating splashing (on solids). For impacts onto liquids, some evidence exists indicating that the splashing threshold is, at most, shifted by changes in the ratio between the liquid and the gas viscosities, effectively introducing a third dimensionless parameter, i.e. $\mu_{l} / \mu_{g}$, (Zhang et al. 2012; Josserand \& Thoroddsen 2016). In this work, we limit our analysis to the classical parametrisation of Bird et al. (2009) and Thoraval et al. (2012). Figure 4 shows the different regimes observed in our experiments, plotted in terms of the dimensionless groups $K=W e \sqrt{R e}$ and $\left(v_{t} / v_{n}\right)(1 / \sqrt{R e})$, where $v_{t}$ is the (tangential) speed of the substrate. Under this parameterisation, the different behaviours group well as in the previous observations of Thoraval et al. (2012) for static pools.

Splashing of drops impacting static thin liquid films has been observed to take place at $K \sim 2000$ by Cossali, Coghe \& Marengo (1997) and Yarin (2006). In contrast, the onset of splashing in our experiments has been pushed to lower values of $K(K=800)$, which might be due to the extra inertia added to the system by the moving liquid target.

\section{Discussion and conclusions}

For practical applications, the diagram in figure 5 could be used to predict the splashing behaviour under most practical conditions. This is potentially critical in industrial environments where the impact conditions could be optimised to enhance mixing by vortex shedding, or adapted to smooth coalescence where good droplet placement is necessary. In addition, the regime map generated here should constitute a valuable tool to validate numerical models under a wide range of parameters.

This work shows that the different behaviours of drops impacting a moving liquid pool are well parameterised by the common splashing parameter $K$ and the dimensionless group proposed by Bird et al. (2009). These combine both the fluid properties and the impact and pool speeds, based on the growth rate of the developing lamella.

Further experimental studies could concentrate on the effect of the ambient pressure and the ratio between liquid and gas viscosity on the impact behaviour, as these factors are known to modify the splashing on stationary substrates (Xu et al. 2005, 2007; Zhang et al. 2012; Riboux \& Gordillo 2014). Such studies could offer additional insight into novel methods to suppress the undesired effects of splashing.

The studies presented in this manuscript were all performed at a pool depth of $6.0 \mathrm{~mm}$ to facilitate the operation of the experimental rig. Preliminary experiments 
showed that the impact behaviour is not affected by the pool depth as long as it is deeper than $5.0 \mathrm{~mm}$. The impact behaviour of fast droplets onto stationary pools is known to depend on the pool depth (as described in Thoraval et al. (2013)) and this effect is a matter for a future work that would require a modified rig.

Additional material and the experimental data are available online at https://doi.org/10.1017/jfm.2016.672.

\section{Acknowledgements}

This project was supported by the EPSRC (grant EP/H018913/1), the John Fell Oxford University Press Research Fund, and the Royal Society (through a University Research Fellowship and a Research Grant).

\section{Supplementary data and movies}

Supplementary data and movies are available at https://doi.org/10.1017/jfm.2016.672.

\section{REFERENCES}

Bird, J. C., Dhiman, R., Kwon, H. \& Varanasi, K. K. 2013 Reducing the contact time of a bouncing drop. Nature 503, 385-388.

Bird, J. C., Tsai, S. S. H. \& Stone, H. A. 2009 Inclined to splash: triggering and inhibiting a splash with tangential velocity. New J. Phys. 11, 063017.

Castrejón-Pita, A. A., Castrejón-Pita, J. R. \& Hutchings, I. M. 2012 Experimental observation of von Kármán vortices during drop impact. Phys. Rev. E 86, 045301.

Castrejón-Pita, J. R., Morrison, N. F., Harlen, O. G., Martin, G. D. \& Hutchings, I. M. 2011 Experiments and Lagrangian simulations on the formation of droplets in drop-on-demand mode. Phys. Rev. E 83, 036306.

Cossali, G. E., Coghe, A. \& Marengo, M. 1997 The impact of a single drop on a wetted solid surface. Exp. Fluids 22, 463-472.

Deegan, R. D., Brunet, P. \& Eggers, J. 2008 Complexities of splashing. Nonlinearity 21, C1-C11.

DERBY, B. 2010 Inkjet printing of functional and structural materials: fluid property requirements, feature stability, and resolution. Annu. Rev. Mater. Res. 40, 395-414.

Eggers, J. \& Villermaux, E. 2008 Physics of liquid jets. Rep. Prog. Phys. 71, 0366601.

Howison, S. D., Ockendon, J. R. \& Oliver, J. M. 2004 Oblique slamming, planing and skimming. J. Engng Maths 48, 321-337.

Josserand, C. \& Thoroddsen, S. T. 2016 Drop impact on a solid surface. Annu. Rev. Fluid Mech. 48, 365-391.

Josserand, C. \& Zaleski, S. 2003 Droplet splashing on a thin liquid film. Phys. Fluids 15, 1650.

Korob Kin, A. A. 1988 Inclined entry of a blunt profile into an ideal fluid. Fluid Dyn. 23, 443-447.

Lhuissier, H., TAgawa, T., Tran, T. \& SUN, C. 2013 Levitation of a drop over a moving surface. J. Fluid Mech. R4, 733.

LiU, Y., TANG, P. \& XU, L. 2015 Kelvin-Helmholtz instability in an ultrathin air film causes drop splashing on smooth surfaces. Proc. Natl Acad. Sci. USA 112, 3280-3284.

Moore, M. R., Howison, S. D., Ockendon, J. R. \& Oliver, J. M. 2012 Three-dimensional oblique water-entry problems at small deadrise angles. J. Fluid Mech. 711, 259-280.

Moore, M. R., Ockendon, H., Ockendon, J. R. \& Oliver, J. M. 2014 Capillary and viscous perturbations to Helmholtz flows. J. Fluid Mech. 742, R1.

Riboux, G. \& Gordillo, J. M. 2014 Experiments of drops impacting a smooth solid surface: a model of the critical impact speed for drop splashing. Phys. Rev. Lett. 113, 024507.

Sun, S. L. \& WU, G. X. 2013 Oblique water-entry of non-axisymmetric bodies at varying speed by a fully nonlinear method. Q. J. Mech. Appl. Maths 66, 366-393. 
Thoraval, M.-J., Takehara, K., Etoh, T. G., Popinet, S., Ray, P., Josserand, C., Zaleski, S. \& Thoroddsen, S. T. 2012 von Kármán vortex street within an impacting drop. Phys. Rev. Lett. 108, 264506.

Thoraval, M.-J., Takehara, K., Etoh, T. G. \& Thoroddsen, S. T. 2013 Drop impact entrapment of bubble rings. J. Fluid Mech. 724, 234-258.

Thoroddsen, S. T. 2002 The ejecta sheet generated by the impact of a drop. J. Fluid Mech. 451, 373-381.

Thoroddsen, S. T., Etoh, T. G. \& Takehara, K. 2008 High-speed imaging of drops and bubbles. Annu. Rev. Fluid Mech. 40, 257-285.

Thoroddsen, S. T. \& Mahadevan, L. 1997 Experimental study of coating flows in a partially-filled horizontally rotating cylinder. Exp. Fluids 23, 1-13.

Worthington, A. M. 1908 A Study of Splashes. Longman, Green and Co.

XU, L., BARcos, L. \& NAGEL, S. R. 2007 Splashing of liquids: interplay of surface roughness with surrounding gas. Phys. Rev. E 76, 066311.

Xu, L., Zhang, W. W. \& Nagel, S. R. 2005 Drop splashing on a dry smooth surface. Phys. Rev. Lett. 94, 184505.

YARIN, A. L. 2006 Drop impact dynamics: splashing, spreading, receding, bouncing. Annu. Rev. Fluid Mech. 38, 159-192.

Zhang, L. V., Toole, J., FezzaA, K. \& Deegan, R. D. 2012 Splashing from drop impact into a deep pool: multiplicity of jets and the failure of conventional scaling. J. Fluid Mech. 703, 402-413. 